\title{
Evolução Clínica Tardia de Pacientes Multiarteriais Tratados por Revascularização Percutânea e Cirúrgica
}

\author{
Marinella Centemero'1, J. Eduardo Sousa ${ }^{1}$, Adib D. Jatene' ${ }^{1}$ Paulo Paredes Paulista ${ }^{1}$, Luis Carlos Bento Souza ${ }^{1}$, \\ Amanda G. M. R. Sousa' ${ }^{1}$, Fausto Feres ${ }^{1}$, Rodolfo Staico', Luiz Alberto Mattos', Alexandre Abizaid', \\ Galo Maldonado', Luiz Fernando Tanajura', Áurea Chaves' ${ }^{1}$ Leandro Lasave ${ }^{1}$
}

\section{RESUMO}

Introdução: Pacientes multiarteriais tratados percutaneamente apresentam resultados semelhantes àqueles submetidos à cirurgia em relação à ocorrência de eventos cardíacos maiores, embora novos procedimentos de revascularização sejam mais freqüentes nos primeiros. Objetivo: Avaliar os resultados a longo prazo da revascularização percutânea com o implante de stents coronários versus a cirurgia no tratamento da doença multiarterial, verificando a incidência de morte, infarto do miocárdio (IAM), acidente vascular encefálico (AVE) e a realização de novas intervenções. Método: Estudo randomizado realizado no período de abril/ 1997 a junho/1998, no qual foram incluídos 66 pacientes multivasculares submetidos ao implante de stents não farmacológicos (33 P) e à cirurgia (33 P). Resultados: Ao final de um período médio de acompanhamento clínico de 8 anos, constatamos que não ocorreram diferenças significativas na sobrevivência livre de eventos cardiovasculares maiores (morte, IAM e AVE), nos 2 grupos: stent $-74 \%$ x cirurgia - 85,1\%; $\mathrm{p}=$ NS. Procedimentos adicionais de revascularização foram mais freqüentes no pacientes tratados com stents, comparativamente àqueles tratados pela cirurgia (respectivamente, $15 \mathrm{P}-45 \%$ × $5 \mathrm{P}-15 \%$; $\mathrm{p}=0,01$ ). Como conseqüência destes resultados, a sobrevivência livre de todos os eventos, inclusive nova revascularização, foi significativamente menor no grupo percutâneo versus o cirúrgico $(49 \% \times 80,5 \% ; p=0,0082)$. Conclusão: A revascularização com o implante de stents não farmacológicos, no tratamento da doença multiarterial, oferece o mesmo grau de proteção contra eventos cardiovasculares maiores, quando comparada à cirurgia. Contudo, a sobrevivência livre de todos os eventos a longo prazo foi significativamente menor nos pacientes tratados percutaneamente, devido à necessidade mais freqüente de novos procedimentos de revascularização.

DESCRITORES: Coronariopatia. Contenedores. Revascularização miocárdica. Procedimentos cirúrgicos cardíacos.

\section{SUMMARY}

Late Clinical Outcome after Coronary Stenting versus Bypass Surgery in Patients with Multivessel Disease

Background: Patients with multivessel disease treated percutaneously have similar outcomes when compared to those submitted to surgical procedures in terms of major cardiac events, although the former require new revascularization procedures more frequently. Objective: To assess longterm clinical outcomes after coronary artery bypass graft $(\mathrm{CABG})$ for the treatment of multivessel disease versus surgical procedures to treat multivessel disease determining death, AMI, and stroke incidence rates, as well as new interventions. Methods: A randomized study carried out between April, 1997 and June, 1998 included 66 patients submitted to nondrug eluting stenting (33P) and surgery (33P). Results: Clinical follow-up results after 8 years have shown no significant difference between the two groups in terms of survival free of MACE events (death, stroke or AMI): STENTING: $74 \% X$ CABG: $85.1 \%$; $p=$ not significant). Additional revascularizations were more frequent in the stenting group as compared to the surgery group $(15 \mathrm{P}-45 \% \times 5 \mathrm{P}-15 \%$, respectively, $\mathrm{p}=0.01$ ). Consequently, survival rates without MACE and repeated revascularization was significantly lower in patients assigned to stenting when compared to those assigned to CABG $(49 \% \times 80.5 \%$, respectively, $p=0.0082)$. Conclusion: Percutaneous revascularization with non-drug eluting stenting for the treatment of multivessel disease offers the same degree of protection against MACE (death, stroke and AMI) as compared to CABG. However, stenting is associated with a greater need of repeated revascularization and lower total event-free survival rates.

DESCRIPTORS: Coronary disease. Stents. Myocardial revascularization. Cardiac surgical procedures.

1 Instituto Dante Pazzanese de Cardiologia, São Paulo, SP. Correspondência: Marinella Centemero. Av. Dr. Dante Pazzanese, 500 - Ibirapuera - São Paulo, SP. CEP 04012-180 - Fone: (011) 5085-4141 - Fax: (011) 5549-7807 - E-mail: mpcentemero@yahoo.com.br Recebido em: 15/01/2007 • Aceito em: 15/02/2007 
$\mathbf{P}$ ortadores de doença coronária multiarterial representam cerca de $60 \%$ dos pacientes candidatos à revascularização miocárdica'. De um modo geral, os tratamentos percutâneo e cirúrgico apresentam resultados equivalentes em termos de ocorrência de morte, infarto do miocárdio (IAM) e acidente vascular encefálico (AVE), na fase hospitalar e na evolução de médio prazo. Entretanto, a necessidade de novos procedimentos de revascularização é, consistentemente, maior naqueles tratados percutaneamente, devido, principalmente, à ocorrência da reestenose coronária no primeiro $\mathrm{ano}^{2,3}$. A evolução do tratamento percutâneo com a substituição dos balões pelas próteses coronárias e a utilização sistemática destas, a partir da segunda metade da década de 90, trouxeram avanços importantes, aprimorando os resultados da técnica ${ }^{4,5}$. O implante dos stents proporciona um excelente resultado angiográfico, garantindo a obtenção de um diâmetro mínimo arterial amplo e homogêneo, o que, associado à antiagregação plaquetária dupla (aspirina + tienopiridínicos), permitiu a diminuição das complicações agudas na fase hospitalar, a elevação dos índices de sucesso e a maior segurança do procedimento. Paralelamente, e em conseqüência destes bons resultados, constatou-se a redução da reestenose coronária e, por conseqüência, da revascularização do vaso-alvo ${ }^{6}$. Estes fatos contribuíram marcantemente para a expansão das indicações da revascularização percutânea no tratamento de portadores de condições clínicas e angiográficas de maior complexidade, como os multiarteriais, tornando esta técnica realmente competitiva quando comparada à cirurgia cardíaca ${ }^{7}$.

Todavia, são escassos os relatos de resultados clínicos a longo prazo após o implante de stents coronários. Os dados disponíveis referem-se, em grande parte, a pesquisas randomizadas e metanálises envolvendo a utilização da angioplastia coronária com os balões $^{8,9}$. Estudos mais recentes como ARTS, SoS e ERACI-II, que empregaram as próteses coronárias de forma sistemática no tratamento da doença multiarterial, apresentaram resultados relativos ao acompanhamento clínico de médio prazo (2 a 5 anos $)^{10,11}$.

Portanto, nesta pesquisa, os autores analisarão os resultados iniciais e, particularmente, a evolução clínica tardia (> 5 anos) de pacientes com acometimento coronário multivascular submetidos ao implante de stents, comparando-os àqueles tratados por revascularização cirúrgica.

\section{OBJETIVO}

Analisar a ocorrência de morte cardíaca, IAM, AVE, além da realização de procedimentos adicionais de revascularização (percutâneos ou cirúrgicos), no primeiro ano de evolução e tardiamente (> 5 anos).

\section{MÉTODO}

No período compreendido entre abril de 1997 e junho de 1998, 66 pacientes foram selecionados, no
Instituto Dante Pazzanese de Cardiologia, para participarem de um estudo internacional, multicêntrico e randomizado (ARTS), cujo objetivo foi comparar o desempenho da cirurgia versus o tratamento percutâneo empregando stents não farmacológicos, em portadores de doença multiarterial ${ }^{7}$.

Verificados os critérios de inclusão e exclusão da pesquisa pelo cardiologista clínico, a cinecoronariografia foi analisada pelo cardiologista intervencionista e pelo cirurgião, que deveriam concordar em relação à obtenção do grau equivalente de revascularização pelas respectivas técnicas. É importante enfatizar que não havia exigência de necessidade de revascularização completa por ambos os métodos.

\section{Seleção dos pacientes}

Foram incluídos os portadores de isquemia silenciosa, angina estável e instável, não previamente submetidos à cirurgia ou à intervenção percutânea, e que apresentassem lesões > 50\%, em pelo menos dois vasos epicárdicos principais, adequados para o implante de stents e para revascularização cirúrgica.

A angioplastia com o balão foi permitida em vasos de pequeno calibre $(<2,75 \mathrm{~mm})$ desde que, pelo menos, outras duas obstruções em artérias diferentes fossem tratadas com as próteses coronárias.

Excluíram-se os portadores de lesões moderadas e graves em tronco de artéria coronária esquerda, aqueles com fração de ejeção inferior a 30\% ou com insuficiência cardíaca, antecedentes de AVE, IAM com supradesnivelamento do segmento ST $<7$ dias, presença de doença hepática ou renal grave, neutropenia e plaquetopenia, além de contra-indicações ao uso de aspirina e ticlopidina. Também foram excluídos os pacientes que necessitassem de correção cirúrgica de outras doenças cardiovasculares como, por exemplo, doenças valvares, aneurismas de ventrículo esquerdo ou de aorta e lesões graves em território carotídeo.

Após a verificação destes critérios e a obtenção da assinatura do termo de consentimento, procedia-se à randomização do tratamento por meio de uma central telefônica.

\section{Tratamento}

Os stents foram implantados de acordo com a prática habitual da época, realizando-se a pós-dilatação com altas pressões. As próteses utilizadas no estudo foram do tipo CROWN ${ }^{\text {TM }}$ e CROSS-FLEX, ambas da CORDIS (Johnson \& Johnson). O tratamento cirúrgico também seguiu a técnica padrão correspondente ao período de realização do estudo, dando-se ênfase ao emprego da artéria mamária interna esquerda para a revascularização da artéria descendente anterior e da cardioplegia fria com cristalóide ou sangue para a proteção miocárdica. 
Centemero M, et al. Evolução Clínica Tardia de Pacientes Multiarteriais Tratados por Revascularização Percutânea e Cirúrgica. Rev Bras Cardiol Invas 2007; 15(1): 35-43.

Todos os pacientes submetidos ao implante de stents foram tratados com aspirina na dose de 100 $200 \mathrm{mg} /$ dia associada à ticlopidina $250 \mathrm{mg}$, a cada 12 horas, pelo menos 24 horas pré-procedimento. Após a intervenção percutânea, a ticlopidina foi mantida na mesma dose, por 30 dias, e a aspirina, indefinidamente.

Os marcadores de lesão miocárdica (CK total e CKMB) foram mensurados nos seguintes períodos: 6, 12 e 18 horas após a realização da intervenção percutânea e da cirurgia. No caso de se verificarem elevações destes marcadores, novas dosagens foram realizadas até que seus valores retornassem aos níveis normais.

\section{Definições}

- Morte Cardíaca - foram consideradas como de origem cardíaca todas as mortes, exceto aquelas comprovadamente de outra origem, de preferência documentadas por autópsia.

- Infarto do Miocárdio - foram considerados 2 tipos:

1) Na $1^{\text {a }}$ semana pós-procedimento: aparecimento de novas ondas $Q$ não presentes no eletrocardiograma prévio (código de Minnesota), associado a alterações das enzimas cardíacas definidas como a razão do pico máximo de CKMB/CK > 10\% ou a elevação da CKMB > 5 vezes o valor normal, presentes em três amostras consecutivas;

2) Após a $1^{\text {a }}$ semana do procedimento: presença de pelo menos duas características como dor precordial típica > 20 minutos de duração; aparecimento de novas ondas Q não presentes no eletrocardiograma prévio (código de Minnesota); alterações das enzimas cardíacas definidas como a razão do pico máximo de CKMB/ CK $>10 \%$ ou a elevação da CKMB $>5$ vezes o valor normal, presentes em três amostras consecutivas.

- Nova Revascularização - todo procedimento de revascularização percutânea ou cirúrgica realizado e sua causa determinante (presença de angina e/ou provas funcionais com evidência de isquemia).

\section{Seguimento clínico}

Os pacientes foram acompanhados no Instituto Dante Pazzanese de Cardiologia por um período médio de 8 anos. As consultas ambulatoriais foram realizadas no $1^{\circ}$ mês pós-procedimento e aos 3, 6 e 12 meses, nas quais realizava-se o controle clínico, de exames laboratoriais e provas funcionais, se necessário. Posteriormente, os pacientes compareciam a cada 6 meses ou, pelo menos, anualmente; na impossibilidade de comparecimento às consultas, o contato foi feito por telefone com o próprio paciente ou seu médico assistente.

\section{Análise estatística}

Variáveis contínuas foram expressas como média e desvio-padrão e comparadas pelo teste T Student; o teste exato de Fischer foi utilizado para variáveis categóricas e as curvas de sobrevivência livres de eventos foram estimadas pelo método de Kaplan-Meier, sendo as diferenças identificadas pelo teste log-rank.

\section{RESULTADOS}

Sessenta e seis pacientes multiarteriais foram recrutados entre abril de 1997 e junho de 1998, no Instituto Dante Pazzanese de Cardiologia (São Paulo, Brasil), para receberem tratamento percutâneo com implante de stents não farmacológicos (33P) e cirurgia de revascularização miocárdica (33P). Nas Tabelas 1 e 2, podemos observar as características clínicas e angiográficas destes dois grupos.

Todos os 66 pacientes receberam o tratamento para o qual foram designados (33 para stent e 33 para cirurgia) e nenhum evento cardiovascular ocorreu após a randomização e antes da realização dos procedimentos percutâneos e cirúrgicos previstos. Da mesma forma, não foram necessárias intervenções de urgência na

TABELA 1

Características demográficas dos $66 \mathrm{P}$ submetidos ao implante de stents e cirurgia

\begin{tabular}{lccc}
\hline & STENT $-\mathbf{3 3} \mathbf{P}$ & CIRURGIA - 33 P & $\mathbf{p}$ \\
\hline Sexo masculino & $25(76 \%)$ & $23(70 \%)$ & $\mathrm{NS}$ \\
Média de idade & 54,9 anos & 55,2 anos & NS \\
Hipertensão arterial & $23(70 \%)$ & $23(70 \%)$ & $\mathrm{NS}$ \\
Dislipidemia & $18(54,5 \%)$ & $25(76,5 \%)$ & $\mathrm{NS}$ \\
Diabete melito & $6(18 \%)$ & $8(24 \%)$ & $\mathrm{NS}$ \\
Tabagismo & $16(48,5 \%)$ & $15(45,5 \%)$ & $\mathrm{NS}$ \\
Antecedente de infarto & $12(36 \%)$ & $19(57,5 \%)$ & $\mathrm{NS}$ \\
Quadro clínico & $7(21 \%)$ & $11(33 \%)$ & $\mathrm{NS}$ \\
$\quad$ Angina estável & $12(36 \%)$ & $10(31 \%)$ & $\mathrm{NS}$ \\
$\quad$ Angina instável & $14(43 \%)$ & $12(36 \%)$ & $\mathrm{NS}$ \\
$\quad$ Isquemia silenciosa & & \\
\hline
\end{tabular}


TABELA 2

Características angiográficas dos $66 \mathrm{P}$ submetidos ao implante de stents e cirurgia

\begin{tabular}{lccc}
\hline & STENT - 33 P & CIRURGIA - 33 P & p \\
\hline № vasos acometidos & & & NS \\
2 vasos & $23(70 \%)$ & $24(73 \%)$ & NS \\
3 vasos & $10(30 \%)$ & $9(27 \%)$ & NS \\
Artéria tratada & & & NS \\
$\quad$ Descendente anterior & $27(40 \%)$ & $29(35 \%)$ & NS \\
$\quad$ Coronária direita & $25(37 \%)$ & $25(30,5 \%)$ & NS \\
Circunflexa & $16(23 \%)$ & $22(27 \%)$ & NS \\
$\quad$ Diagonal & 0 & $6(7,5 \%)$ & $56,5 \%$ \\
Fração de ejeção & $56,2 \%$ & & \\
\hline
\end{tabular}

fase hospitalar, após a realização dos tratamentos, em ambos os grupos.

As características técnicas das intervenções estão descritas na Tabela 3 .

Qualquer elevação dos marcadores de lesão miocárdica foi identificada mais freqüentemente no grupo cirúrgico comparativamente ao percutâneo (31 P 94\% x 11 P - 33\%, p=0,0001). Quando consideramos elevações da CKMB acima de 5 vezes o limite superior do valor normal, estas ocorreram em $2(6 \%)$ pacientes tratados por stent e em 5 (15\%) tratados cirurgicamente $(p=N S)$. Não ocorreram casos de trombose aguda e subaguda do stent, nem tampouco infecções relacionadas à cirurgia. Entretanto, no grupo cirúrgico, um paciente apresentou AVE no pós-operatório, comprovado por exame neurológico e tomografia de crânio.

Os resultados das intervenções percutânea e cirúrgica na fase hospitalar estão discriminados na Tabela 4.

Os 66 pacientes incluídos neste estudo foram acompanhados tardiamente em nosso ambulatório. Dos 33

TABELA 3

Características técnicas dos procedimentos percutâneo e cirúrgico

\begin{tabular}{lcc}
\hline & STENT - 33 P & CIRURGIA - 33 P \\
\hline № stents implantados / paciente & 2,4 & - \\
№ enxertos realizados / paciente & - & 2,5 \\
\% P com mamária esquerda & $17 \mathrm{~mm}$ & $27(82 \%)$ \\
Média da extensão dos stents & $33(100 \%)$ & \\
Sucesso da intervenção percutânea & & \\
\hline
\end{tabular}

TABELA 4

Resultados hospitalares dos $66 \mathrm{P}$ submetidos ao implante de stents e cirurgia

\begin{tabular}{|c|c|c|c|}
\hline & STENT - 33 P & CIRURGIA - 33 P & $\mathbf{p}$ \\
\hline Morte & 0 & 0 & NS \\
\hline IAM com presença de onda Q & 0 & $1(3 \%)$ & NS \\
\hline AVE & 0 & $1(3 \%)$ & NS \\
\hline Cirurgia de urgência & 0 & 0 & NS \\
\hline Infecção pós-operatória & - & 0 & NS \\
\hline Outras complicações & $2(6 \%) *$ & $4(12 \%)^{* *}$ & NS \\
\hline
\end{tabular}


Centemero M, et al. Evolução Clínica Tardia de Pacientes Multiarteriais Tratados por Revascularização Percutânea e Cirúrgica. Rev Bras Cardiol Invas 2007; 15(1): 35-43.

pertencentes ao grupo tratado pelo implante de stents, 29 (88\%) completaram o período de 8 anos; apenas $4(12 \%)$ não o fizeram, mas foram seguidos por, pelo menos, 5 anos (exigência do estudo). No grupo cirúrgico, 26 (79\%) pacientes realizaram o seguimento tardio de 8 anos, 3 (9\%) completaram o período mínimo de 5 anos e $4(12 \%)$ foram seguidos por, pelo menos, 3 anos, sendo perdidos após.

No Gráfico 1-A e na Figura 1-A, observa-se a evolução clínica ao final do primeiro ano de tratamento, a qual revela taxas reduzidas de eventos adversos e curvas de sobrevivência livres de eventos cardiovasculares maiores (morte, IAM e AVE), semelhantes nos pacientes submetidos à revascularização percutânea e cirúrgica. Quando consideramos também a necessidade de procedimentos adicionais de revascularização, nos primeiros 12 meses, verificamos uma diferença entre os 2 grupos, favorecendo o desempenho daqueles tratados cirurgicamente (Gráfico 1-A e Figura 1-B). Nos pacientes submetidos ao implante de stents coronários, a realização de nova intervenção neste período deveu-se, principalmente, à ocorrência da reestenose e foi necessária em $7 \mathrm{P}(21 \%)$, sendo 3 tratados por meio de nova angioplastia (43\%) e 4 (57\%) por cirurgia. Apenas um (3\%) caso de IAM sem supradesnivelamento do segmento ST ocorreu em paciente que apresentava

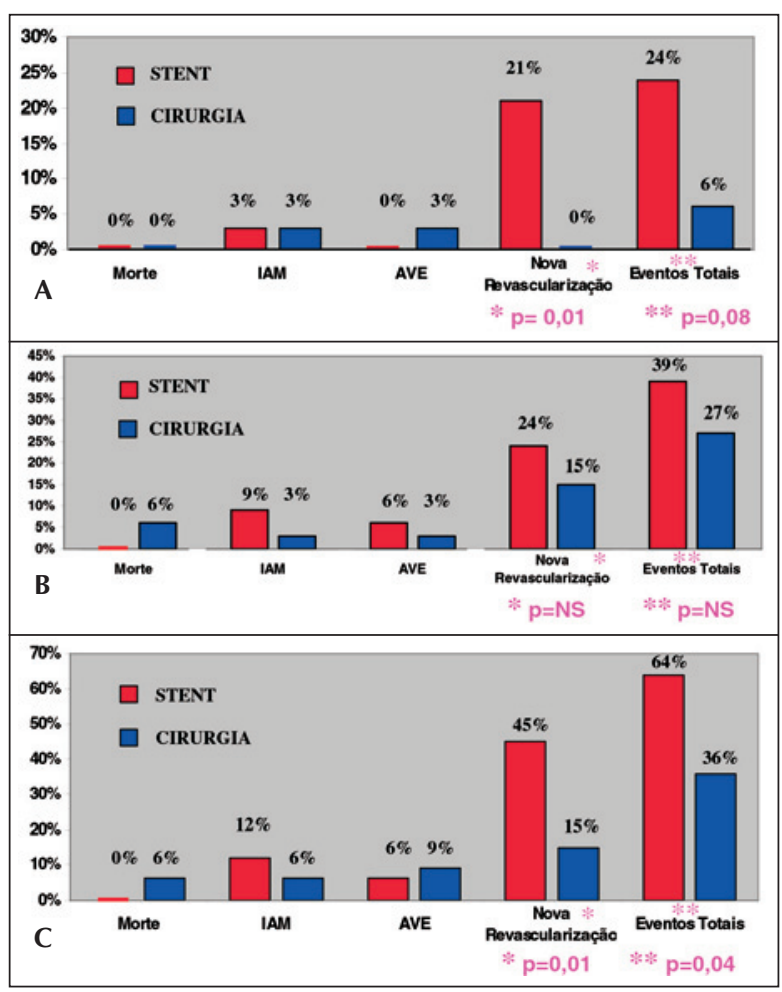

Gráfico 1 - Eventos cardiovasculares maiores e nova revascularização nos pacientes submetidos ao implante de stent e à cirurgia. A: no $1^{\circ}$ ano; B: após o $1^{\circ}$ ano; C: período completo (desde a realização do procedimento até o último contato obtido). reestenose coronária, a qual foi tratada pelo implante de novo stent.

No Gráfico 1-B, podemos verificar que, após o primeiro ano de evolução, as taxas de mortalidade, IAM, AVE e, também, de nova revascularização são similares nos pacientes tratados por stent e cirurgia.

Os mesmos eventos cardíacos e cerebrovasculares são analisados no Gráfico 1-C, agora considerando o tempo total de evolução, desde a realização dos procedimentos percutâneos e cirúrgicos até o último contato obtido com o paciente. Observa-se, neste caso, que a incidência de nova revascularização foi significativamente maior no grupo percutâneo, perfazendo um total de eventos adversos superior àquele encontrado no grupo cirúrgico.

Por sua vez, as curvas de sobrevivência livres de eventos cardiovasculares maiores, num período de acompanhamento médio de 8 anos, revelaram resultados semelhantes nos 2 grupos (Figura 1-C). Contudo, persiste a diferença significativa em relação à realização de novas intervenções, mais freqüentes nos pacientes tratados percutaneamente com o implante de stents comparativamente à cirurgia (Figura 1-D). Como podemos observar, a divergência entre as 2 curvas inicia-se no primeiro ano de evolução e mantém-se no período tardio.

\section{DISCUSSÃO}

Neste estudo, verificamos que os pacientes multiarteriais submetidos à revascularização percutânea (com implante de stents não farmacológicos) e cirúrgica apresentam resultados clínicos de boa qualidade e semeIhantes quanto à ocorrência de morte, IAM e AVE, num período de acompanhamento médio de 8 anos. A diferença observada entre os 2 tratamentos está relacionada à realização de novos procedimentos de revascularização, que são mais freqüentes no grupo submetido ao implante de stents, devido à ocorrência da reestenose no primeiro ano e tardiamente relacionados à progressão da aterosclerose coronária em outros territórios.

\section{Resultados precoces / primeiro ano}

Em pacientes multiarteriais, com perfil clínico e angiográfico de moderado risco, caso da nossa pesquisa, as taxas de sucesso do procedimento percutâneo na fase hospitalar foram elevadas, com ausência de complicações maiores. Também não foram identificados casos de trombose da prótese no primeiro mês pós-intervenção. É importante ressaltar que estes resultados excelentes foram obtidos num período no qual a importância do tratamento antiplaquetário adjunto ainda não era devidamente valorizada ${ }^{12,13}$. Atualmente, várias pesquisas revelam que a utilização precoce (pelo menos 24 horas antes do implante) e em altas doses dos tienopiridínicos, em especial do clopidogrel, conferem, 


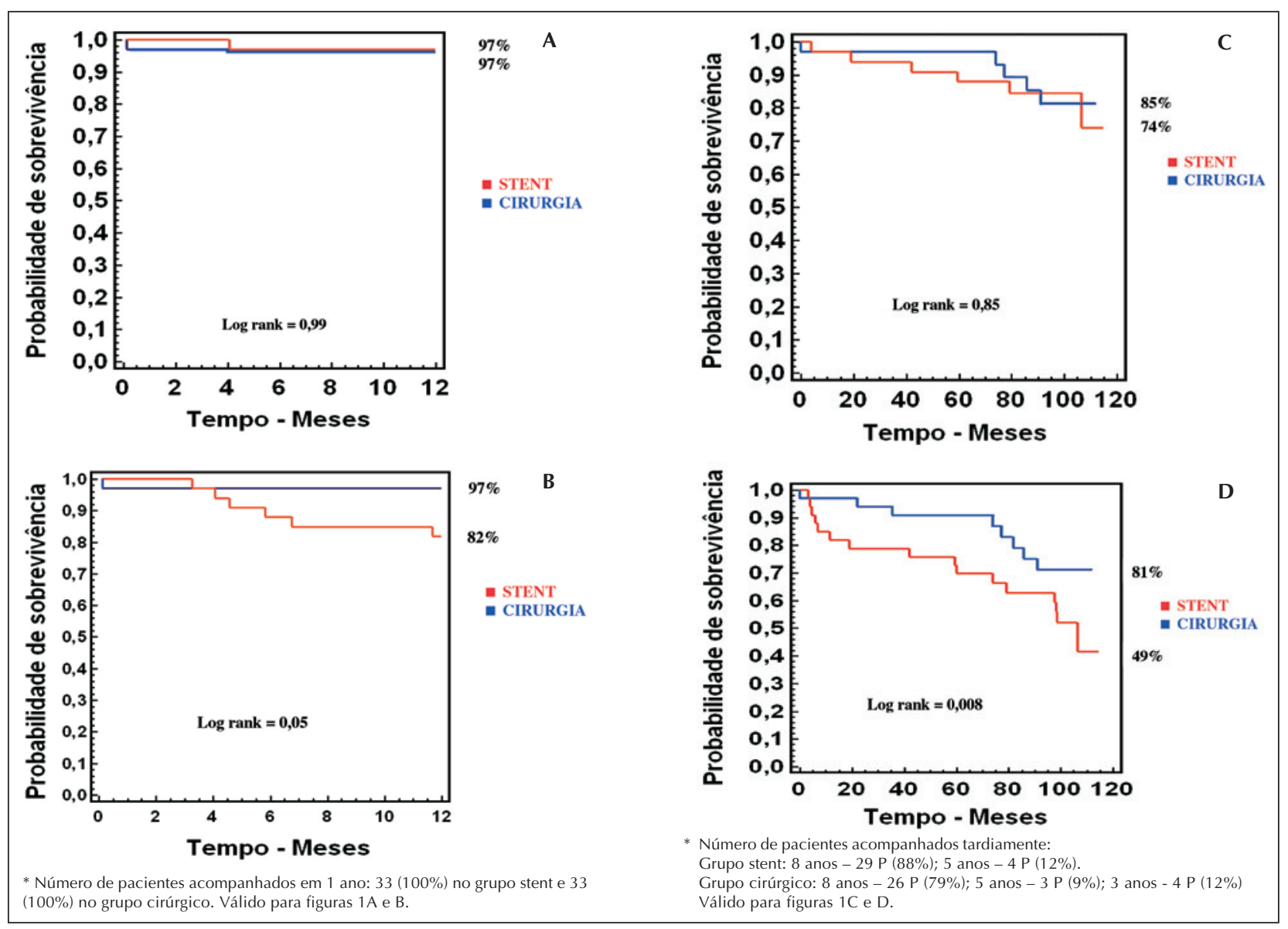

Figura 1 - Curvas de sobrevivência livres de eventos cardiovasculares nos 66 pacientes submetidos ao implante de stents e à cirurgia. A: Curvas de sobrevivência livres de morte, IAM e AVE - 1ํano; B: Curvas de sobrevivência livres de morte, IAM e AVE + nova revascularização - 1ㅇano; C: Curvas de sobrevivência livres de morte, IAM e AVE - tardio; D: Curvas de sobrevivência livres de morte, IAM e AVE + nova revascularização - tardio.

juntamente com os beta-bloqueadores e os inibidores das glicoproteínas IIb / IIla em situações específicas, proteção à circulação coronária distal, reduzindo as microembolizações da placa ateromatosa durante o procedimento e, por conseguinte, a liberação dos marcadores de lesão miocárdica'14-16. De fato, a elevação das enzimas cardíacas em valores muito acima daqueles considerados normais (> 5 vezes) ocorreu numa minoria de pacientes submetidos à revascularização percutânea $(6 \%)$ e num porcentual inferior àquele encontrado no grupo cirúrgico (15\%). Algumas pesquisas relacionam este fenômeno a resultados clínicos insatisfatórios, constituindo-se em fator preditor com impacto negativo na sobrevivência tardia, inclusive nos pacientes tratados por cirurgia ${ }^{17,18}$.

Na comparação entre as duas técnicas também é interessante observar que o grau de revascularização obtido por ambas foi equivalente, visto que foram implantados, em média, 2,4 stents/paciente e realizados 2,5 enxertos/paciente, no grupo cirúrgico. Vale ressaltar que a maioria dos pacientes tratados em nosso estudo apresentava doença coronária biarterial.
Quanto à revascularização cirúrgica, os resultados imediatos foram os esperados para este grupo: baixos índices de complicações cardiovasculares e ausência de infecções no pós-operatório, mesmo em pacientes diabéticos $(24 \%)$, reflexo da excelência da técnica, dos cuidados intensivos imediatos e da seleção adequada dos pacientes. Enfatizamos a importância da utilização freqüente da artéria mamária interna esquerda para a revascularização preferencial do território da descendente anterior ( $82 \%$ dos casos). As altas taxas de permeabilidade deste enxerto propiciam um maior grau de proteção da parede anterior à eventual progressão da doença aterosclerótica, além de desempenhar papel relevante como fonte de circulação colateral para outras regiões miocárdicas em risco ${ }^{19}$.

No primeiro ano pós-revascularização, constatamos que ambos os tratamentos conferem o mesmo grau de proteção em relação à ocorrência de morte, IAM e AVE, fato este corroborado por vários estudos randomizados e séries consecutivas que compararam a intervenção percutânea utilizando balões ou stents x cirurgia ${ }^{10,20}$. 
Centemero M, et al. Evolução Clínica Tardia de Pacientes Multiarteriais Tratados por Revascularização Percutânea e Cirúrgica. Rev Bras Cardiol Invas 2007; 15(1): 35-43.

Neste período, novas intervenções ocorreram apenas nos pacientes tratados percutaneamente, e sua causa foi a presença da reestenose coronária. Este fenômeno repercute nas curvas de sobrevivência livre de todos os eventos cardiovasculares, nas quais verificamos uma diferença de 15 pontos percentuais entre os dois tratamentos. Tal fato já era esperado, na medida em que as próteses coronárias reduziram de forma expressiva a reestenose, embora não tenham eliminado o problema definitivamente ${ }^{6}$. Confrontando estes achados àqueles dos estudos que compararam a angioplastia com o balão x cirurgia, nos quais a necessidade de uma nova intervenção variou de 30\% a 60\%, constatamos uma importante redução desta, o que atenuou a diferença entre as duas técnicas de revascularização $\mathrm{O}^{3,21,22}$.

\section{Resultados a longo prazo}

Tardiamente, novos eventos adversos foram identificados nos pacientes tratados por stent e cirurgia em porcentuais semelhantes e compatíveis àqueles relatados na literatura ${ }^{9}$. Após o primeiro ano, procedimentos adicionais de revascularização devido à progressão da doença coronária ocorreram em ambos os grupos, sem diferença significativa (stent: 8 P - 24\% x cirurgia: $5 \mathrm{P}-15 \%$ ), ainda que em maior número nos pacientes tratados percutaneamente. Nos 8 pacientes deste grupo, novo implante de stent foi realizado em $5(62,5 \%)$, ao passo que, no grupo cirúrgico, todos os 5 pacientes foram tratados com próteses coronárias.

Ao analisarmos o período completo do acompanhamento clínico dos 66 pacientes incluídos neste estudo, desde a realização dos respectivos procedimentos até a data do último contato, nos deparamos novamente com baixas taxas de mortalidade (variando de $0 \%$ para os stents a, aproximadamente, 1\%/ano para a cirurgia), infarto do miocárdio e acidente vascular cerebral (0,5 a $1 \%$ / ano), sem diferenças entre os dois tratamentos. Dos 33 pacientes submetidos ao implante de stents, 15 (45\%) necessitaram nova revascularização, sendo que 8 foram novamente tratados por intervenção percutânea. Nos 33 pacientes submetidos à cirurgia, a progressão da aterosclerose coronária ocorreu em $5(15 \%)$ e todos foram tratados pelo implante de stents. Desta forma, embora a curva de sobrevivência livre de eventos maiores e nova revascularização ao final de 8 anos seja significativamente menor nos pacientes tratados percutaneamente quando comparada à cirurgia $(49 \%$ x 81\%), gostaríamos de chamar a atenção para alguns fatos:

- apenas 7 (21\%) pacientes do grupo percutâneo foram encaminhados à cirurgia no decorrer de 8 anos, ou seja, 79\% permaneceram livres deste tratamento;

- por outro lado, no grupo cirúrgico, nenhum paciente foi submetido à segunda cirurgia, notoriamente associada a um maior risco, pois todos foram tratados percutaneamente;
- por fim, a necessidade de novas intervenções para o tratamento da reestenose ou da progressão da doença arterial coronária não apresentou impacto negativo tardio nas taxas de mortalidade, infarto do miocárdio e acidente vascular encefálico nos dois grupos.

Apesar do número pequeno de pacientes em cada grupo, os resultados desta pesquisa estão em perfeita consonância com aqueles publicados pelo grupo de investigadores do estudo ARTS (que envolveu 1205 pacientes), ao final de 5 anos de acompanhamento clínico ${ }^{23}$.

\section{Novas perspectivas}

É importante ter em mente que a progressão da aterosclerose coronária ainda é um problema sem solução satisfatória à vista, comprometendo a evolução clínica tardia dos portadores desta doença, quer sejam abordados por intervenção percutânea ou cirurgia.

A magnitude do problema deve estimular os cardiologistas e os pacientes na busca do controle rigoroso e efetivo dos fatores de risco tradicionalmente relacionados à doença, particularmente do diabetes, da dislipidemia e do tabagismo. Paralelamente, pesquisas recentes buscam novos marcadores bioquímicos e genéticos que possam auxiliar na estratificação precoce do risco de desenvolvimento de eventos maiores ${ }^{24}$.

Do ponto de vista da farmacologia, novos antiplaquetários e anticoagulantes, além de medicamentos para o tratamento da síndrome metabólica e do diabetes, têm contribuído para a redução da morbi-mortalidade relacionada à aterosclerose coronária ${ }^{25,26}$.

No que diz respeito à intervenção percutânea, o desenvolvimento e a incorporação de novas tecnologias, como os stents com eluição de medicamentos e o estudo in vivo das placas ateromatosas coronárias por meio de ultra-sonografia, da histologia virtual e da tomografia por coerência óptica, já demonstram grandes benefícios. A redução indiscutível das taxas de reestenose e da revascularização do vaso-alvo obtida pela utilização dos stents farmacológicos, como demonstrado pelos estudos FIM, RAVEL, série SIRIUS, RESEARCH, ARTS II e vários outros, expandiu ainda mais as indicações do tratamento percutâneo ${ }^{27-32}$. A segurança e a eficácia da técnica, aliadas à crescente experiência e ousadia dos pesquisadores, têm alargado as fronteiras deste tratamento para subgrupos de altíssimo risco, como os portadores de diabetes associado à doença multivascular e aqueles com lesão de tronco de artéria coronária esquerda não protegido. Estudos que pesquisam especificamente estas questões estão em andamento e seus resultados, certamente, trarão novos e importantes ensinamentos a respeito da doença coronária ${ }^{33-37}$.

\section{REFERÊNCIAS BIBLIOGRÁFICAS}

1. Rigter H, Meijler AP, McDonnell J, Scholma JK, Bernstein SJ. Indications for coronary revascularisation: a Dutch perspective. Heart 1997;77:211-8. 
2. The Bypass Angioplasty Revascularization Investigation (BARI) Investigators. Comparison of coronary bypass surgery with angioplasty in patients with multivessel disease. N Engl J Med 1996;335:217-25.

3. Sim I, Gupta M, McDonald K, Bourassa MG, Hlatky MA. A meta-analysis of randomized trials comparing coronary artery bypass grafting with percutaneous transluminal coronary angioplasty in multivessel coronary artery disease. Am J Cardiol 1995;76:1025-9.

4. Fischman DL, Leon MB, Baim DS, Schatz RA, Savage MP, Penn I, et al. A randomized comparison of coronary-stent placement and balloon angioplasty in the treatment of coronary artery disease. Stent Restenosis Study Investigators. N Engl J Med 1994;331:496-501.

5. Serruys PW, Jaegere P, Kiemeneij F, Macaya C, Rutsch W, Heyndrickx G, et al. A comparison of balloon-expandablestent implantation with balloon angioplasty in patients with coronary artery disease. N Engl J Med 1994;331:489-95.

6. Serruys PW, van Hout B, Bounnier H, Legrand V, Garcia E, Macaya C, et al. Randomised comparison of implantation of heparin-coated stents with balloon angioplasty in selected patients with coronary artery disease (Benestent II). Lancet 1998;352:673-81.

7. Serruys PW, Unger F, Sousa JE, Jatene A, Bonnier HJ, Schonberger JP, et al. Comparison of coronary-artery bypass surgery and stenting for the treatment of multivessel disease. N Engl J Med 2001;344:1117-24.

8. Seven-year outcome in the Bypass Angioplasty Revascularization Investigation (BARI) by treatment and diabetic status. J Am Coll Cardiol 2000;35:1122-9.

9. Hoffman SN, TenBrook JA, Wolf MP, Pauker SG, Salem DN, Wong JB. A meta-analysis of randomized controlled trials comparing coronary artery bypass graft with percutaneous transluminal coronary angioplasty: one-to eight- year outcomes. J Am Coll Cardiol 2003;41:1293-304.

10. SoS Investigators. Coronary artery bypass surgery versus percutaneous coronary intervention with stent implantation in patients with multivessel coronary artery disease (the Stent or Surgery trial): a randomised controlled trial. Lancet 2002;360:695-70.

11. Rodriguez AE, Baldi J, Fernandez-Pereira C, Navia J, RodriguezAlemparte M, Delacasa A, et al. Five-year follow-up of the Argentine randomized trial of coronary angioplasty with stenting versus coronary bypass surgery in patients with multiple vessel disease (ERACI II). J Am Coll Cardiol 2005; 46:582-8.

12. Yusuf S, Zhao F, Mehta SR, Chrolavicius S, Tognoni G, Fox KK. Effects of clopidogrel in addition to aspirin in patients with acute coronary syndromes without ST-segment elevation. Clopidogrel in Unstable Angina to Prevent Recurrent Events Trial Investigators. N Engl J Med 2001;345:494-502.

13. Steinhubl SR, Berger PB, Mann JT $3^{\text {rd }}$, Fry ET, DeLago O, Wilmer $\mathrm{C}$, et al. Early and sustained dual oral antiplatelet therapy following percutaneous coronary intervention: a randomized controlled trial. JAMA 2002;288:2411-20.

14. Boersma E, Akkerhuis KM, Theroux P, Califf RM, Topol EJ, Simoons ML. Platelet glycoprotein IIb/IIla receptor inhibition in non-ST-elevation acute coronary syndromes: early benefit during medical treatment only, with additional protection during percutaneous coronary intervention. Circulation 1999;100:2045-8.

15. Karvouni E, Katritsis DG, loannidis JP. Intravenous glycoprotein $\mathrm{Ilb} / \mathrm{Ill}$ a receptor antagonists reduce mortality after percutaneous coronary interventions. J Am Coll Cardiol 2003;41:26-32.

16. Sharma SK, Kini A, Marmur JD, Fuster V. Cardioprotective effect of prior beta-blocker therapy in reducing creatine
kinase-MB elevation after coronary intervention: benefit is extended to improvement in intermediate-term survival. Circulation 2000;102:166-72.

17. Abdelmeguid AE, Topol EJ. The myth of the myocardial 'infarctlet' during percutaneous coronary revascularization procedures. Circulation 1996;94:3369-75.

18. Saucedo J, Popma J, Mehran R, Bucher T, Abizaid AS, Curran M. Lack of association of intermediate CPK-MB elevation and late mortality in patients treated with intracoronary stents. J Am Coll Cardiol 2002;31:215.

19. Brooks MM, Jones RH, Bach RG, Chaitman BR, Kern JM, Orszulak TA. Predictors of mortality and mortality from cardiac causes in the bypass angioplasty revascularization investigation (BARI) Randomized trial and registry. Circulation 2000;101:2682-9.

20. Rodriguez A, Bernardi V, Navia J, Baldi J, Grinfeld L, Martinez J, et al. Argentine Randomized Study: Coronary Angioplasty with Stenting versus Coronary Bypass Surgery in patients with Multiple-Vessel Disease (ERACI II): 30-day and one-year follow-up results. J Am Coll Cardiol 2001;37:51-8.

21. CABRI Trial Participants. First-year results of CABRI (Coronary Angioplasty versus Bypass Revascularisation Investigation). Lancet 1995;346:1179-84.

22. King SB 3rd, Lembo NJ, Weintraub WS, Kosinski A, Barnhart HX, Kutner MH, et al. Emory Angioplasty versus Surgery Trial (EAST). N Engl J Med 1994;331:1044-50.

23. Serruys PW, Ong AT, van Herwerden LA, Sousa JE, Jatene A, Bonnier JJ, et al. Five-year outcomes after coronary stenting versus bypass surgery for the treatment of multivessel disease: the final analysis of the Arterial Revascularization Therapies Study (ARTS) randomized trial. J Am Coll Cardiol 2005;46:575-81.

24. Morrow DA. Preprocedural C-reactive protein for risk prediction before percutaneous coronary intervention ( $\mathrm{PCl}$ ): a US perspective. Clin Chem 2004;50:1489-91.

25. Bhatt DL, Fox KA, Hacke W, Berger PB, Black $H$, Boden $W E$, et al. Clopidogrel and aspirin versus aspirin alone for the prevention of atherothrombotic events. N Engl J Med 2006;354:1706-17.

26. Nathan DM. Clinical practice. Initial management of glycemia in type 2 diabetes mellitus. N Engl J Med 2002;347:1342-9.

27. Sousa JE, Costa MA, Abizaid A, Abizaid AS, Feres F, Pinto $I M$, et al. Lack of neointimal proliferation after implantation of sirolimus-coated stents in human coronary arteries: a quantitative coronary angiography and three-dimensional intravascular ultrasound study. Circulation 2001;103:192-5.

28. Morice MC, Serruys PW, Sousa JE, Fajadet J, Ban Hayashi E, Perin $M$, et al. A Randomized comparison of a sirolimuseluting stent with a standard stent for coronary revascularization. N Engl J Med 2002;346:1773-80.

29. Moses JW, Leon MB, Popma JJ, Fitzgerald PJ, Holmes DR, $\mathrm{O}^{\prime}$ Shaughnessy $\mathrm{C}$, et al. Sirolimus-eluting stents versus standard stents in patients with stenosis in a native coronary artery. N Engl J Med 2003;349:1315-23.

30. Schofer J, Schluter M, Gershlick AH, Wijns W, Garcia E, Schampaert E, et al. Sirolimus-eluting stents for treatment of patients with long atherosclerotic lesions in small coronary arteries: double-blind,randomised controlled trial (E-SIRIUS). Lancet 2003;362:1093-9.

31. Lemos PA, Serruys PW, van Domburg RT, Saia F, Arampatzis CA, Hoye A, et al. Unrestricted utilization of sirolimuseluting stents compared with conventional bare stent implantation in the "real world": the Rapamycin-Eluting Stent Evaluated At Rotterdam Cardiology Hospital (RESEARCH) registry. Circulation 2004;109:190-5. 
Centemero M, et al. Evolução Clínica Tardia de Pacientes Multiarteriais Tratados por Revascularização Percutânea e Cirúrgica. Rev Bras Cardiol Invas 2007; 15(1): 35-43.

32. Serruys PW, Lemos PA, van Hout BA. Sirolimus eluting stent implantation for patients with multivessel disease: rationale for the Arterial Revascularisation Therapies Study part II (ARTS II). Arterial Revascularisation Therapies Study part II Steering Committee and Investigators. Heart 2004;90:995-8.

33. Moussa I, Leon MB, Baim DS, O'Neill WW, Popma J, Buchbinder $M$, et al. Impact of sirolimus-eluting stent on outcome in diabetic patients: a SIRIUS (SIRollmUS-coated Bx Velocity ballon-expandable stent in the treatment of patients with de novo coronary lesions) substudy. Circulation 2004;109:2273-8.

34. Sobel BE, Frye R, Detre KM. Bypass Angioplasty Revascularization Investigation 2 Diabetes Trial. Burgeoning dilemmas in the management of diabetes and cardiovascular disease: rationale for the Bypass Angioplasty Revascularization
Investigation 2 Diabetes (BARI 2D) trial. Circulation 2003; 107:636-42.

35. Flaherty JD, Davidson CJ. Diabetes and coronary revascularization. JAMA 2005;293:1501-8.

36. Elsasser A, Mollmann H, Nef HM, Hamm CW. How to revascularize patients with diabetes mellitus: Bypass or stents and drugs? Clin Res Cardiol 2006;95:195-203.

37. Valgimigli M, Malagutti $P$, Rodriguez-Granillo GA, Tsuchida K, Garcia-Garcia HM, van Mieghem CA, et al. Single-vesse versus bifurcation stenting for the treatment of distal left main coronary artery disease in the drug-eluting stenting era. Clinical an angiographic insights into the Rapamycin-Eluting Stent Evaluated at Rotterdam Cardiology Hospital (RESEARCH) and Taxus-Stent Evaluated at Rotterdam Cardiology Hospital (T-SEARCH) registries. Am Heart J 2006;152:896-902. 